\title{
Empirical lead generation by crystallographic screening of fragment libraries
}

\author{
Debanu Das \\ Accelero Biostructures, San Francisco, CA \\ www.accelerobio.com \\ info@accelerobio.com
}

Fragment-based drug discovery (FBDD) is widely used in the pharmaceutical industry to provide novel leads for developing new therapeutics. It allows a more efficient scanning of chemical space with a higher hit rate compared to high-throughput screening, and this has important outcomes in early drug discovery and can be especially relevant for challenging non-druggable targets. FBDD has resulted in 30 new drugs entering clinical trials with 2 that have entered the market.

X-ray crystallography is the gold standard for determining the exact binding orientation of a fragment as an essential step in this process. Absence of crystal structures of the target with bound fragments is a significant impediment in FBDD. Conventional crystallography is inefficient for screening a large fragment library due to expense and effort. Complementary techniques, such as Surface Plasmon Resonance, Thermal Shift Assay or Nuclear Magnetic Resonance are often used to prescreen for fragments that bind, while protein crystallography is used in a second step to determine the exact binding of each fragment. Absence of crystals structures of drug targets bound to fragments is a significant impediment to medicinal chemistry optimization of the fragment hits.

With foundational expertise in structural genomics and high-throughput protein X-ray crystallography, we have developed novel capabilities to counter the above bottlenecks in early drug discovery. Our platform for crystallography-based fragment screening accelerates the development of lead compounds by directly providing 3D structures of fragments bound to a target of interest, potentially leading to $\sim 5-7 x$ reduction in early drug discovery times. This provides unprecedented experimental route to high quality, high reliability and high value results simultaneously: identification and assessment of fragment binding sites and poses; target ligandability; and differentiation of orthosteric and allosteric sites and binders. We will present results from applying our platform to different classes of drug targets. 